\title{
Virtual Wayfinding: A Comparison of Orientation and Navigation Behavior in Built and Virtual Environments
}

\author{
María Paz Campos \\ Universidad de Chile | Chile | maria.campos.1@ug.uchile.cl \\ Tannia Basaure \\ Universidad de Chile | Chile | tannia.basaure@ug.uchile.cl \\ Mauricio Loyola \\ Universidad de Chile | Chile | mloyola@uchile.cl
}

\begin{abstract}
In this study we compare the wayfinding behavior of occupants in virtual reality and built spaces. Results show significant differences related to the speed of navigation, geometry of movement, and frequency of stops and turns. Subjective evaluations of spatial characteristics show no significant differences between built and virtual spaces.
\end{abstract}

Keywords: Virtual Reality, Head-Mounted Display, Wayfinding, Spatial Navigation, Occupant Behavior,

\section{INTRODUCCIÓN}

En los últimos años se ha observado un crecimiento exponencial del uso de la realidad virtual (RV) en visualización arquitectónica motivada por la mayor disponibilidad y accesibilidad de equipos comerciales de tipo head-mounted displays (HMD). Consecuentemente, las aplicaciones de la tecnología han ido progresivamente evolucionando desde la simple visualización estática de espacios virtuales durante el proceso de diseño a usos dinámicos e interactivos, tales como evaluación de legibilidad espacial, simulación de uso, o evaluación del comportamiento de navegación y orientación (wayfinding).

Sin embargo, existen numerosos estudios que sugieren que existen diferencias sustanciales en la percepción espacial y dimensional estática de los usuarios entre entornos virtuales y construidos (Claessen et al 2016, Heydarian et al 2015, Kimura et al 2017, Kuliga et al 2015) y, por lo tanto, es razonable sospechar que también estas diferencias también podrían existir en experiencias espaciales dinámicas como wayfinding. Si estas diferencias fueran sustantivas, la precisión representacional y capacidad predictiva de las simulaciones en RV quedarían cuestionadas.

Esta investigación contribuye a dilucidar este asunto por medio de una comparación experimental del comportamiento de navegación y orientación de personas en espacios virtuales y construidos equivalentes que aporte evidencia empírica para estimar y reflexionar sobre las posibilidades y limitaciones del uso de la realidad virtual como herramienta de representación y/o simulación del comportamiento de las personas.

\section{METODOS}

El experimento consistió en observar el comportamiento de navegación y orientación de un grupo de personas en dos situaciones: en un espacio construido tradicional (real) y en un espacio equivalente modelado digitalmente (virtual), mientras resolvía una tarea espacial sencilla. Las variables observadas incluyeron aspectos estáticos y dinámicos de la navegación (recorrido, duración, velocidad), acciones corporales (detenerse, girar, agacharse, etc.), comprensión y percepción espacial (lumínica, acústica y sensación "térmica"), y aspectos generales de la propia experiencia de uso de equipos HMD.

El espacio construido usado escenario para el experimento fue el cuarto nivel de la biblioteca de la Facultad de Economía y Negocios de la Universidad de Chile. Este espacio ofrecía buena legibilidad espacial dada por su configuración sencilla, alta variabilidad de recorrido (i.e. diversas opciones de generar una ruta a su interior) y posibilidad de generar distintas tareas espaciales equivalentes en complejidad (e.g. buscar un objeto en el espacio). El espacio virtual consistió en una réplica idéntica del espacio desarrollada con ArchiCAD y Unreal Engine. La Figura 1 muestra una fotografía del espacio real y una imagen renderizada del espacio virtual. La Figura 2 muestra la planta general del espacio.

La muestra estuvo constituida por 54 estudiantes de pregrado, en su gran mayoría estudiantes de arquitectura, reclutados de manera abierta, por conveniencia, a través de anuncios públicos e invitaciones en talleres de arquitectura. Entre los criterios de selección para los participantes se incluyó tener familiaridad con tecnologías digitales CAD y 3D (y por consiguiente, capacidad mínima de abstracción con modelos digitales) y no conocer el espacio previamente. 

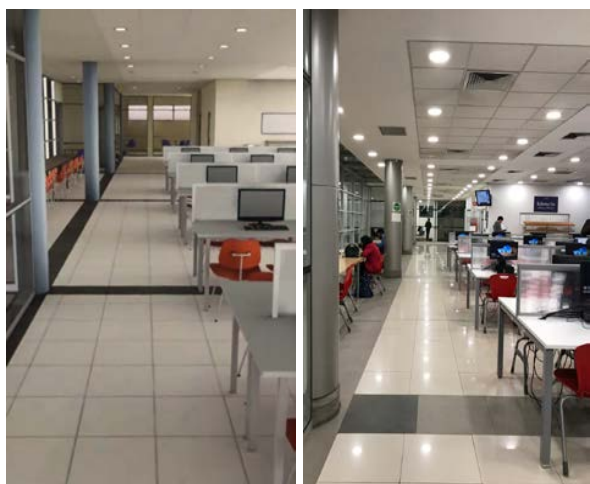

Figura 1: Izq.: Espacio virtual. Der.: Espacio construido

Los participantes fueron aleatoriamente divididos en 2 grupos. El primer grupo recorrió el espacio construido primero y luego el espacio virtual con RV. El segundo grupo lo hizo al revés.: el espacio virtual primero, y luego el construido.

A cada participante se le solicitó recorrer el espacio (virtual y construido) hasta encontrar 2 cubos de colores azul y amarillo ubicadas en alguna sala o rincón del espacio. La ubicación de los cubos fue diferente entre el espacio virtual y construido, pero con igual nivel de complejidad de localización (Figura 2). Todos los participantes iniciaron su recorrido en el mismo punto (caja de escaleras central) y se les solicitó volver a ese punto de origen una vez que hayan encontrado ambos cubos.

En el recorrido en el espacio construido, los participantes portaban una cámara GoPro a la altura de la cabeza que registró sus movimientos. Para el recorrido en el espacio virtual se utilizaron equipos Oculus Rift CV1, y se grabó un video de la visualización y se registraron todos los movimientos (posición y tiempo) del avatar (Figura 3)

Al término de ambos recorridos, todos los participantes fueron entrevistados y respondieron una encuesta sobre comprensión y percepción espacial en ambos espacios, y sobre la experiencia de uso de equipos RV.

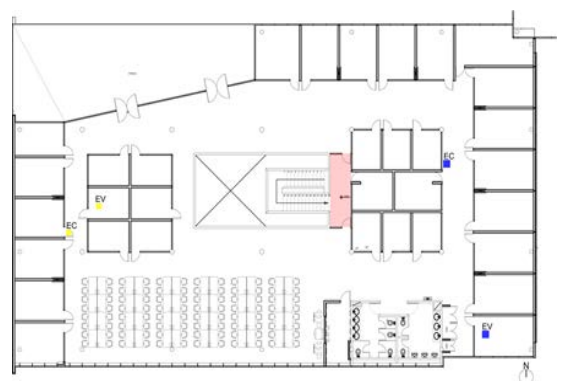

Figura 2: Ubicación de cubos (amarillo y azul) y origen (rosado).

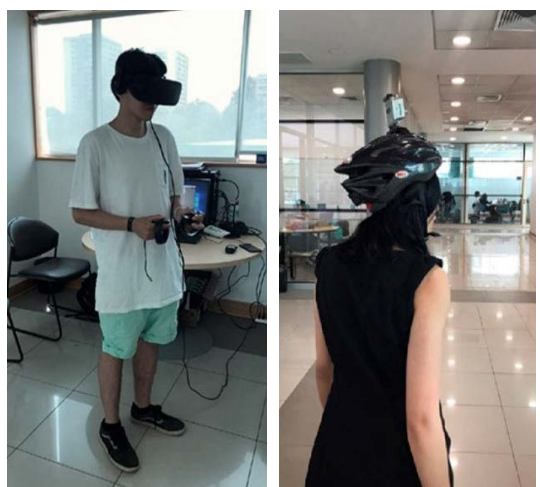

Figura 3: Izq. Participante en espacio virtual. Der: Participante en espacio construido.

La entrevista y encuesta incluyeron 4 secciones. La primera, sobre percepción de distancias y tiempo de recorrido (Tabla 1). La segunda, sobre percepción acústica, lumínica y "sensación térmica", evaluadas usando escalas de Likert semánticas de 5 niveles (Tabla 2). La tercera sección incluyó preguntas abiertas para obtener impresiones cualitativas más profundas sobre la experiencia (Tabla 3). Por último, la cuarta sección abordó preguntas generales de la experiencia de uso de equipos $\mathrm{RV}$, tales como molestias causadas por la interfaz y opiniones personales sobre la actividad.

Tabla 1: Preguntas de percepción de distancias

\begin{tabular}{l}
\hline ¿Cuánta distancia existe... \\
\hline ...entre el origen y el primer cubo? \\
...entre el primer y segundo cubo? \\
...entre el segundo cubo y el origen? \\
\hline ¿Cuánto tiempo demoraste en... \\
\hline$\ldots$ encontrar el primer cubo (desde el origen)? \\
... encontrar el segundo cubo (desde el primer cubo)? \\
\hline
\end{tabular}

Tabla 2: Preguntas de percepción espacial

\begin{tabular}{|c|c|c|c|c|}
\hline \multicolumn{5}{|c|}{ Sensaciones acústicas } \\
\hline $\begin{array}{l}\text { Muy } \\
\text { ruidoso }\end{array}$ & Ruidoso & $\begin{array}{l}\mathrm{Ni} \\
\text { ruidoso ni } \\
\text { silencioso }\end{array}$ & Silencioso & $\begin{array}{c}\text { Muy } \\
\text { silencioso }\end{array}$ \\
\hline \multicolumn{5}{|c|}{ Sensaciones lumínicas } \\
\hline $\begin{array}{c}\text { Muy } \\
\text { oscuro }\end{array}$ & Oscuro & $\begin{array}{l}\text { Ni oscuro } \\
\text { ni } \\
\text { luminoso }\end{array}$ & Luminoso & $\begin{array}{c}\text { Muy } \\
\text { luminoso }\end{array}$ \\
\hline \multicolumn{5}{|c|}{ Sensación térmica } \\
\hline Muy frío & Frío & $\begin{array}{l}\text { Ni frío ni } \\
\text { caluroso }\end{array}$ & Caluroso & $\begin{array}{c}\text { Muy } \\
\text { caluroso }\end{array}$ \\
\hline
\end{tabular}


Tabla 3: Preguntas abiertas

Preguntas abiertas
¿Dónde fue más fácil y difícil orientarte? ¿Por qué?
Describe la sensación de presencia percibida dentro del
espacio virtual
¿Cuáles fueron las diferencias percibidas más significativas
entre el espacio virtual y el espacio construido?
¿Crees que la realidad virtual es un buen o mal método para
la representación de la navegación de espacios
construidos? ¿Por qué?

\section{RESULTADOS}

Las Figuras 4 a 9 muestran representaciones gráficas de los análisis dinámicos y estáticos.

Las figuras 4 y 5 muestran las trayectorias (recorridos) en los espacios virtual y construido, respectivamente. Se puede apreciar que la navegación en el espacio virtual fue más extendida y dispersa espacialmente, mientras que en el espacio construido fue más directa y rectilínea. Se vislumbra aquí una de las primeras diferencias entre ambas experiencias: en el espacio virtual, los participantes exploraron el espacio fundamentalmente a través del movimiento, mientras que, en el espacio construido, la exploración también se realizó a través de la mirada.

Las figuras 6 y 7 muestran en análisis de densidad de flujo (cantidad total de personas por unidad de superficie), representados en escala de color (cian, menos densidad; rojo, más densidad). En el espacio virtual, el flujo fue similar en todo el espacio salvo en los dos puntos donde se encontraban los objetos a encontrar. En el espacio construido, la densidad muestra claramente que las personas prefirieron caminar por el eje central de los corredores, distanciados de los muros laterales (excepto, obviamente, en los puntos de búsqueda).

Las figuras 8 y 9 muestran el análisis estático detallando acciones corporales distintas al desplazamiento (detención, observación, giro, agacharse, etc.). Mientras que las miradas hacia ambos lados fueron equivalentes en cantidad y ubicación en ambos espacios, las detenciones y giros fueron significativamente más frecuentes en el espacio virtual. Los participantes debieron detenerse y girar para mirar constantemente para orientarse y asegurarse de estar cubriendo efectivamente todo el territorio de búsqueda. De hecho, ningún participante realizó un giro completo $\left(360^{\circ}\right)$ en el espacio construido, mientras que en el espacio virtual eso ocurrió 40 veces. Notablemente, en el espacio construido las personas también se agacharon muchísimo más que en el espacio virtual, lo que podría estar asociado a limitaciones de usabilidad intuitiva de la interfaz HMD.

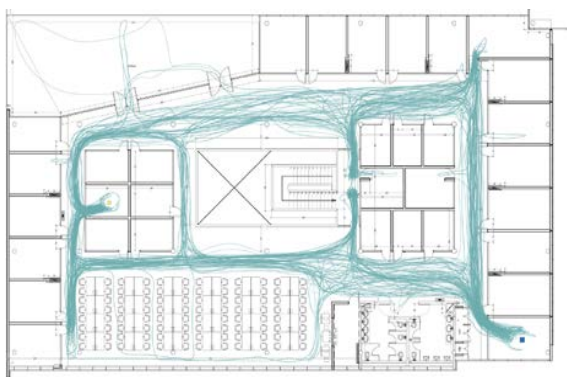

Figura 4: Análisis de trayectoria en espacio virtual.

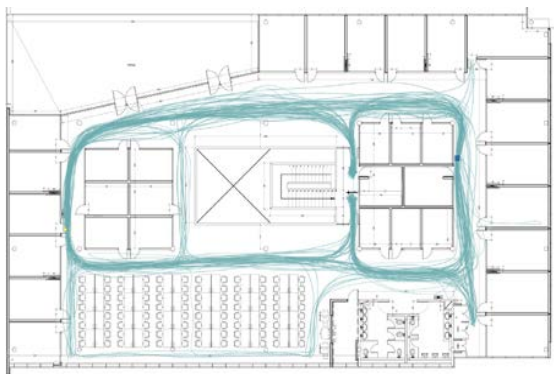

Figura 5: Análisis de trayectoria en espacio construido.

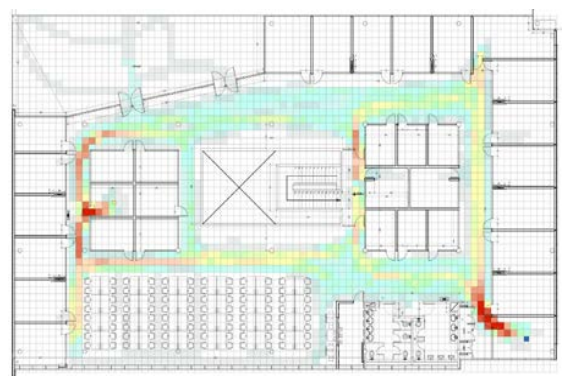

Figura 6: Análisis de flujo en espacio virtual.

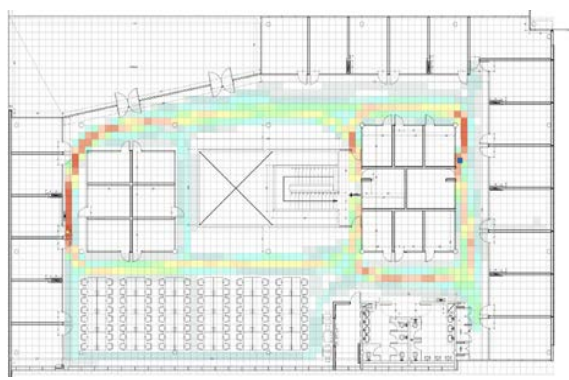

Figura 7: Análisis de flujo en espacio construido. 


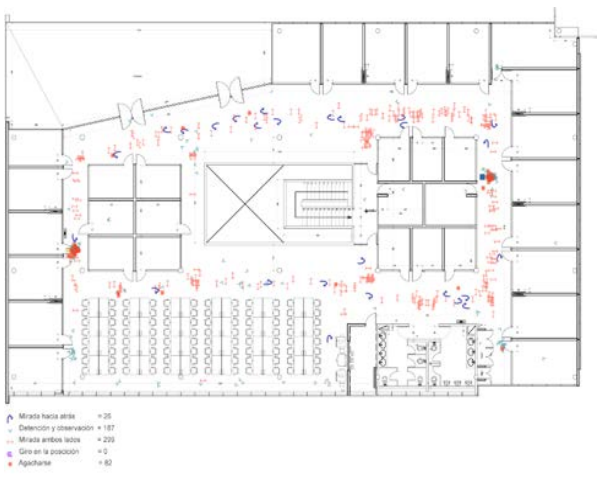

Figura 8: Análisis estático en espacio construido.

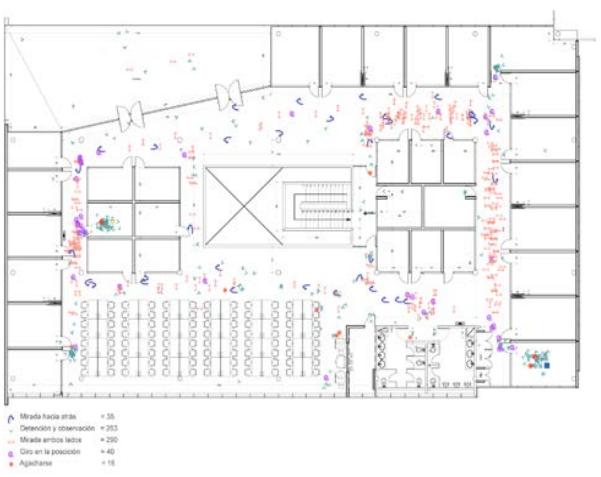

Figura 9: Análisis estático en espacio virtual.

Las Tablas 4 y 5 muestran los resultados de distancia, tiempo y velocidad efectivas y percibidas.

Tabla 4: Tiempo y distancia promedio percibida y real en la navegación en espacio virtual y construido.

\begin{tabular}{lcccc}
\hline Distancia & \multicolumn{2}{c}{ Espacio Virtual } & \multicolumn{2}{c}{ Espacio construido } \\
$(\mathrm{m})$ & Percibido & Real & Percibido & Real \\
\hline Tramo 1 & 14.1 & 17.5 & 15.8 & 21.0 \\
Tramo 2 & 23.3 & 33.5 & 22.6 & 30.5 \\
Tramo 3 & 14.3 & 17.5 & 11.4 & 10.0 \\
Total & 51.8 & 68.5 & 49.7 & 61.5 \\
\hline Tiempo & \multicolumn{2}{c}{ Espacio Virtual } & Espacio construido \\
(s) & Percibido & Real & Percibido & Real \\
\hline Tramo 1 & 133 & 68 & 65 & 34 \\
Tramo 2 & 160 & 104 & 115 & 59 \\
Tramo 3 & 89 & 63 & 67 & 27 \\
Total & 382 & 235 & 247 & 117 \\
\hline
\end{tabular}

Consistente con lo reportado en la literatura, la estimación de distancia fue inferior a la real en ambos espacios, pero con diferencias más marcadas en el espacio virtual $(-24.3 \%)$. Un fenómeno similar se observa en la estimación de tiempo: fue sobrestimada en ambos espacios, pero con mayor distorsión en el espacio virtual.
El tiempo total de navegación en el espacio virtual fue notablemente superior al tiempo de navegación en el espacio construido, lo que representa una diferencia importante considerando que las condiciones espaciales y la complejidad de las tareas de búsqueda eran equivalentes en ambos casos. La velocidad promedio de recorrido total en el espacio virtual fue $0.31 \mathrm{~m} / \mathrm{s}$ mientras que en el espacio construido alcanzó $0.52 \mathrm{~m} / \mathrm{s}$, consistente con una caminata muy lenta. En el espacio construido se aprecia también una leve aceleración durante el ejercicio, pasando de $0.46 \mathrm{~m} / \mathrm{s}$ promedio en el primer tramo, a 0.57 $\mathrm{m} / \mathrm{s}$ promedio en el último tramo. Este fenómeno no se observó en el espacio virtual, donde los participantes mantuvieron velocidades relativamente constantes durante todo el ejercicio.

La evaluación de percepciones espaciales arrojó una mezcla de resultados predecibles y otros sorpresivos. La sensación acústica fue muchísimo más silenciosa en la experiencia virtual; resultado previsible considerando que el modelo RV no incluía ruido ambiente. Las sensaciones "térmicas" no mostraron tendencia. Como referencia, investigaciones anteriores han obtenido diferencias en la percepción de cualidades espaciales no visuales (incluyendo sensación de temperatura ambiente) en entornos RV. Sin embargo, en la percepción lumínica es donde se encontraron los resultados más sorpresivos. La inmensa mayoría de los participantes (88.9\%) consideraron el espacio virtual "luminoso" o "muy luminoso", muy por sobre la cantidad de participantes que asignaron esos calificativos al espacio construido. Cabe recordar, que el modelo digital fue construido y renderizado con un nivel de iluminancia similar al espacio construido (quizás incluso levemente disminuido, ver Figura 1).

Al comparar la sensación general de facilidad de orientación y navegación, la tendencia fue absoluta. El $76 \%$ de los participantes consideró que fue más fácil orientarse en el espacio construido; el 18.5\% consideró ambos espacios completamente equivalentes; y apenas un $5.6 \%$ consideró más fácil orientarse en el espacio virtual.

Respecto a la evaluación general de la experiencia, y en particular, las molestias ocasionadas por los equipos RV, el principal aspecto mencionado fue mareo, calificado por un $37 \%$ de los participantes en nivel 4 en una escala 1 a 5 ( $1=\sin$ molestias, $5=$ muchas molestias). Mucho más atrás le siguen las menciones a descoordinación leve en la navegación (nivel 2). Esto probablemente se explica por la falta de familiaridad con la interfaz de navegación de los equipos HMD. Otras molestias no recibieron menciones a niveles significativos.

A pesar de las molestias, la gran mayoría de los participantes calificó la actividad positivamente. Destacaron las cualidades "entretenida", "agradable", "atractiva", "limpio", y "coherente [entre virtual y construido]". Respecto de esto último, un $51.9 \%$ de los participantes evaluó la similitud entre la experiencia espacial virtual y construida en un nivel 4 , en una escala de 1 a 5 ( 1 =muy diferente, $5=$ =muy idéntico).

En el análisis cualitativo de las entrevistas dos temas asomaron como muy relevantes: la importancia sustantiva del propio cuerpo en la percepción espacial del movimiento 
(propiocepción dinámica) y la (no) inclusión de personas en el entorno virtual como factor perjudicial para la sensación de presencia.

La falta de percepción del propio cuerpo humano, o al menos de un avatar que replicara los movimientos corporales de los participantes fue sindicado como un aspecto limitante del espacio virtual para la percepción especial, y por oposición, como un factor ventajoso en el espacio construido. Por ejemplo, algunas opiniones de participantes son:

“[Orientarse] fue más fácil en el espacio construido, creo que fue por el hecho de que en este podía usar todos mis sentidos y mi cuerpo requería coordinación y movimiento para encontrar los cubos."

"Me siento más familiarizada con el espacio construido ya que distingo las proporciones en relación a mi cuerpo."

"En el espacio virtual] me sentía ajena al no ver mis extremidades, no podía sentirme parte del lugar."

"A pesar del parecido del espacio virtual al espacio construido, es difícil sentirse realmente dentro del lugar. El movimiento es distinto y las proporciones son más difíciles de percibir al no tener un cuerpo para comparar."

Un segundo aspecto destacado por los participantes fue la ausencia de otras personas y sonido ambiente humano en el espacio virtual, que produce un efecto de tranquilidad excesiva e irreal, al punto que socava la sensación de presencia. Este fenómeno, denominado "efecto de pueblo fantasma" ya ha sido reportado por los autores en otros estudios similares (XXXXXXXX, 2019). Algunas opiniones de participantes son que demuestran este efecto son:

"Se sintió como un sueño, ya que fuera de la biblioteca todo era blanco, la iluminación era muy plana y no sentí ningún sonido. Además, no se sentía real al estar solo."

"Da la sensación de estar flotando en el espacio."

"Se percibía bastante soledad en el espacio virtual, hasta el punto de ser algo inquietante. La movilidad también jugó un rol importante, los movimientos eran algo limitados. Generaba frustración el no estar $100 \%$ coordinado con los controles, y la descoordinación producía bastante mareo al transitar."

"[Orientarse] fue más fácil en el espacio virtual ya que no había distracciones como personas, sonidos, etc. Además, el contexto al ser neutro concentraba la atención solo en la arquitectura."

Por último, un $91 \%$ de los participantes aprueban la tecnología como método de visualización arquitectónica dadas sus cualidades para replicar la espacialidad, proporciones y materialidad del espacio construido.

\section{DISCUSIÓN}

Los resultados muestran que, en general, el comportamiento de orientación y navegación en espacios virtuales es relativamente similar al observado en espacios construidos, pero con algunas importantes diferencias significativas.

El análisis dinámico de trayectoria muestra que la navegación en el espacio virtual fue más extensa, dispersa y más lenta que en el espacio construido, y el análisis estático de orientación muestra que en el espacio virtual las detenciones, giros y cambios de altura fueron más frecuentes que en el espacio construido. El sentido, secuencia y trazado del recorrido fueron notablemente similares en ambos casos, aunque ligeramente más rectilíneo en el espacio construido. En otras palabras, los resultados sugieren que la aproximación intuitiva a la navegación espacial en espacios virtuales es similar, o al menos comparable a la navegación en espacios construidos, aunque menos espontánea y eficiente.

Al parecer, la poca familiaridad de los participantes con equipos HMD ralentizaron su velocidad y eficiencia de navegación natural. Para descubrir y reconocer el espacio virtual, los participantes detuvieron su recorrido, giraron su cuerpo, y observaron alrededor lenta y conscientemente. En el espacio construido, el descubrimiento y reconocimiento se realizaba de manera inconsciente durante la marcha, rápidamente con leves movimientos de cabeza y (presumiblemente) visión periférica.

Notablemente, el reporte de sensaciones espaciales percibidas fue equivalente entre el espacio virtual y el construido, y la medición de comprensión espacial arrojó resultados parecidos. Las diferencias observadas no son distintas de lo ya reportado en la literatura para modelos estáticos. En este sentido, el nivel de fidelidad y riqueza sensorial del modelo virtual resulta crucial para lograr sensaciones de presencia verosímiles. La inclusión de ruido ambiente apareció como un factor imprescindible para lograr la mayor amplitud y profundidad sensorial necesaria para lograr una alta sensación de presencia. Igualmente, la falta de personas y de las sombras y reflejos que se producen por sus movimientos, parece ser la causa de la sensación de mayor luminosidad y "limpieza visual" del modelo virtual.

Aun así, la mayoría de los participantes señaló que la realidad virtual permitió generar una alta sensación de presencia inmersiva, y catalogaron a la tecnología como una herramienta muy útil para representar espacios arquitectónicos virtualmente de manera fidedigna. Las principales distorsiones parecen provenir de la poca naturalidad del movimiento, por la inhabilitación de las capacidades propioceptivas, y por falta de personas en el modelo virtual, todos asuntos que pueden ser mejorados con mejores interfaces de navegación y modelos RV más completos. 


\section{CONCLUSIÓN}

Este estudio comparó de manera experimental el comportamiento de orientación y navegación de personas en espacios construidos y espacios virtuales visualizados con equipos de realidad virtual de tupo HMD. Las variables observadas incluyeron aspectos estáticos y dinámicos de la navegación, acciones corporales, comprensión y percepción espacial, y aspectos generales de la propia experiencia de uso de equipos HMD, configurando una evaluación cuantitativa y cualitativa completa e integral.

Los resultados demuestran que existen diferencias distinguibles en el comportamiento de orientación y navegación entre espacios virtuales y espacios construidos. En términos generales, la navegación en el espacio virtual fue más extensa, dispersa y más lenta que en el espacio construido, y con mayor cantidad de detenciones y giros para descubrir y reconocer el espacio. Al parecer, estas diferencias derivan de la poca familiaridad de los usuarios con los equipos HMD y la poca naturalidad de la interfaz, especialmente la falta de autopercepción en el avatar y la inhabilitación de las capacidades propioceptivas.

No obstante, los resultados también sugieren que las diferencias no parecen ser distorsiones capitales que sentencien a muerte las posibilidades de la RV para simular comportamientos dinámicos de usuarios en entornos virtuales. Mas bien, se trata de tener precaución al momento de utilizar la tecnología para estudios que exijan precisión en la simulación del comportamiento de navegación de personas y considerar las limitantes y complejidades que fueron identificadas en este trabajo.

Mas aún, la tecnología es atractiva y bien recibida por los usuarios, y es evaluada positivamente como una herramienta potencialmente muy útil para representar espacios arquitectónicos virtualmente de manera fidedigna. Se recomienda, entonces, continuar la investigación en la caracterización y corrección de las distorsiones y así, contribuir al desarrollo de una herramienta eficaz y confiable de simulación y predicción del comportamiento espacial de personas para el diseño arquitectónico.

\section{AGRADECIMIENTOS}

Agradecemos a la Biblioteca de la Facultad de Economía y Negocios de la Universidad de Chile y a sus funcionarios por facilitar las instalaciones para la realización de esta investigación. Agradecemos también al Prof. Bruno Rossi y a Alejandro Flores por su ayuda con los modelos y dispositivos de RV.

\section{REFERENCIAS}

Claessen, M. H., Visser-Meily, J. M., de Rooij, N. K., Postma, A., \& van der Ham, I. J. (2016). A direct comparison of real-world and virtual navigation performance in chronic stroke patients. Journal of the International Neuropsychological Society, 22(4), 467-477.

Heydarian, A., Carneiro, J. P., Gerber, D., Becerik-Gerber, B. Hayes, T., \& Wood, W. (2015). Immersive virtual environments versus physical built environments: A benchmarking study for building design and user-built environment explorations. Automation in Construction, 54, 116-126.

Kimura, K., Reichert, J. F., Olson, A., Pouya, O. R., Wang, X, Moussavi, Z., \& Kelly, D. M. (2017). Orientation in virtual reality does not fully measure up to the real-world. Scientific reports, $7(1), 1-8$

Kuliga, S. F., Thrash, T., Dalton, R. C., \& Hölscher, C. (2015). Virtual reality as an empirical research tool - Exploring user experience in a real building and a corresponding virtual model. Computers, environment and urban systems, 54, 363375 . 